\title{
Regulation of M-Type Potassium Current by Intracellular Nucleotide Phosphates
}

\author{
Mark A. Simmons and Carla R. Schneider \\ The Neuropharmacology Laboratory, Department of Pharmacology, Marshall University, \\ Huntington, West Virginia 25704-9388
}

\begin{abstract}
The effects of intracellular application of various concentrations of adenine nucleoside phosphates and nucleotide analogs on the M-type $\mathrm{K}$ current $\left(I_{\mathrm{M}}\right)$ of single neurons isolated from sympathetic ganglia were studied. With $1 \mathrm{~mm}$ MgATP intracellularly $I_{M}$ decreased to $25 \%$ of its initial level 39 min after the start of whole-cell recording. In the absence of ATP the current decreased more rapidly. Addition of glucose and pyruvate extracellularly was equivalent to adding $1 \mathrm{~mm}$ MgATP intracellularly. AMP-PNP, a nonhydrolyzable ATP analog, at a concentration of 1 or $3 \mathrm{~mm}$ was unable to maintain $I_{M}$ in the absence of ATP. When ATP and AMP-PNP were combined in the pipette, however, the maintenance of $I_{M}$ was prolonged. A series of nucleotides and analogs have been combined with ATP to test for their ability to maintain $I_{M}$ and to alter calcineurin phosphatase activity. There was a positive correlation between the ability of
\end{abstract}

a nucleotide to prevent the rundown of $I_{M}$ and its ability to inhibit calcineurin phosphatase activity. These findings show that the amplitude of $I_{\mathrm{M}}$ is dually regulated by cellular levels of adenine nucleotide diphosphates and triphosphates. A hydrolyzable form of ATP is necessary to maintain the M current. The maintenance of $I_{M}$ is further enhanced by the simultaneous presence of ADP or other adenine nucleotides that alter calcineurin activity, but not by higher concentrations of ATP alone. These results are consistent with regulation of $I_{M}$ by phosphorylation events that maintain $I_{M}$ and dephosphorylation events that lead to current rundown.

Key words: ATP; M-current; sympathetic neuron; potassium current; calcineurin; nucleotide phosphates; phosphorylation; phosphatase
The M-current $\left(I_{\mathrm{M}}\right)$ is a voltage- and time-dependent potassium current that has been observed in a variety of neuronal cell types. $I_{\mathrm{M}}$ was first recorded from sympathetic neurons (Brown and Adams, 1980) and has subsequently been observed in recordings from brain (Halliwell and Adams, 1982; Halliwell, 1986), spinal cord (Murase et al., 1986), and smooth muscle (Sims et al., 1985). $I_{\mathrm{M}}$ is activated at membrane potentials positive to $-60 \mathrm{mV}$ and consists of an outward $\mathrm{K}$ current that does not inactivate. Compounds that act as agonists at gonadotropin-releasing hormone receptors, muscarinic receptors, substance $\mathrm{P}$ receptors, or purinergic receptors inhibit $I_{\mathrm{M}}$. The precise signal transduction mechanism by which these compounds decrease $I_{M}$ is not known, but the data clearly suggest that the inhibition of $I_{M}$ by these receptors follows activation of guanine nucleotide-binding proteins (Pfaffinger, 1988; Brown et al., 1989; Lopez and Adams, 1989; Simmons and Mather, 1992).

Intracellular levels of ATP have been suggested to be involved in the control of $I_{\mathrm{M}}$; however, the nature of this regulation remains unclear. There are conflicting findings with regard to the role of intracellular ATP in modulating $I_{\mathrm{M}}$. Pfaffinger (1988) found that when $I_{M}$ was recorded with electrodes containing a solution that lacked ATP, $I_{\mathrm{M}}$ declined much more rapidly than if the solution suffusing the cell contained ATP. When ATP was replaced by AMP-PNP, the M-current was lost. Tokimasa and Akasu (1990) found that $I_{M}$ in dorsal root ganglion cells disap-

\footnotetext{
Received April 22, 1998; revised June 5, 1998; accepted June 8, 1998.

This work was supported by Grant NS-25999 from the National Institute of Neurological Disorders and Stroke. We thank Dr. James B. Becker and Mr. Robert J. Mather for participating in some of the early experiments.

Correspondence should be addressed to Dr. Mark A. Simmons, Department of Pharmacology, Marshall University, Huntington, WV 25704-9388.

Copyright (C) 1998 Society for Neuroscience 0270-6474/98/186254-07\$05.00/0
}

peared or was reduced to $20 \%$ of its initial value after $15 \mathrm{~min}$ when ATP was replaced by AMP-PNP in the whole-cell recording electrode. Our results (Simmons et al., 1990), however, showed that when intracellular ATP was replaced by AMP-PNP, the current was maintained at $84 \%$ of the initial value after 13 min. Chen and Smith (1992) also found that $I_{M}$ was not affected when ATP was left out of the pipette solution and that AMP-PCP did not affect the rundown of $I_{\mathrm{M}}$.

Direct comparisons of previous studies on $I_{\mathrm{M}}$ are difficult, because different laboratories have used different solutions, both intracellularly and extracellularly. Of particular concern are the concentrations of $\mathrm{MgCl}_{2}, \mathrm{ATP}$, and GTP added to pipette solutions and the presence or absence of metabolic substrates such as glucose and pyruvate in the extracellular solutions and in the pipette. Here, we examine the role of intracellular ATP, a variety of other nucleotide phosphate analogs, and extracellular pyruvate and glucose in the maintenance of $I_{\mathrm{M}}$ in single neurons.

\section{MATERIALS AND METHODS}

Cell isolation. Bullfrogs (Rana catesbeiana) were obtained from Charles D. Sullivan Co. (Nashville, TN) and handled according to national and institutional guidelines with the approval of the Institutional Animal Use and Care Committee. To obtain sympathetic neurons, a frog was chilled in ice for $1 \mathrm{hr}$, rapidly decapitated, and double-pithed. Sympathetic ganglia were dissected and incubated for $90 \mathrm{~min}$ in dissociation medium with collagenase A $(1 \mathrm{mg} / \mathrm{ml})$ and trypsin $(0.5 \mathrm{mg} / \mathrm{ml})$, for $90 \mathrm{~min}$ in dissociation medium with collagenase $\mathrm{B}(0.75 \mathrm{mg} / \mathrm{ml})$, and then stored in growth medium at $4^{\circ} \mathrm{C}$ for up to $3 \mathrm{~d}$. On the day of the experiment, one or two ganglia at a time were triturated 25-50 times with a long-shanked glass Pasteur pipette to free single cells from the ganglia. The cells were placed in a Petri dish on the stage of an inverted microscope and continuously perfused with extracellular solution.

Whole-cell recordings. All experiments were conducted at room temperature, $\sim 20^{\circ} \mathrm{C}$. Whole-cell recordings (Hamill et al., 1981) were made 
A
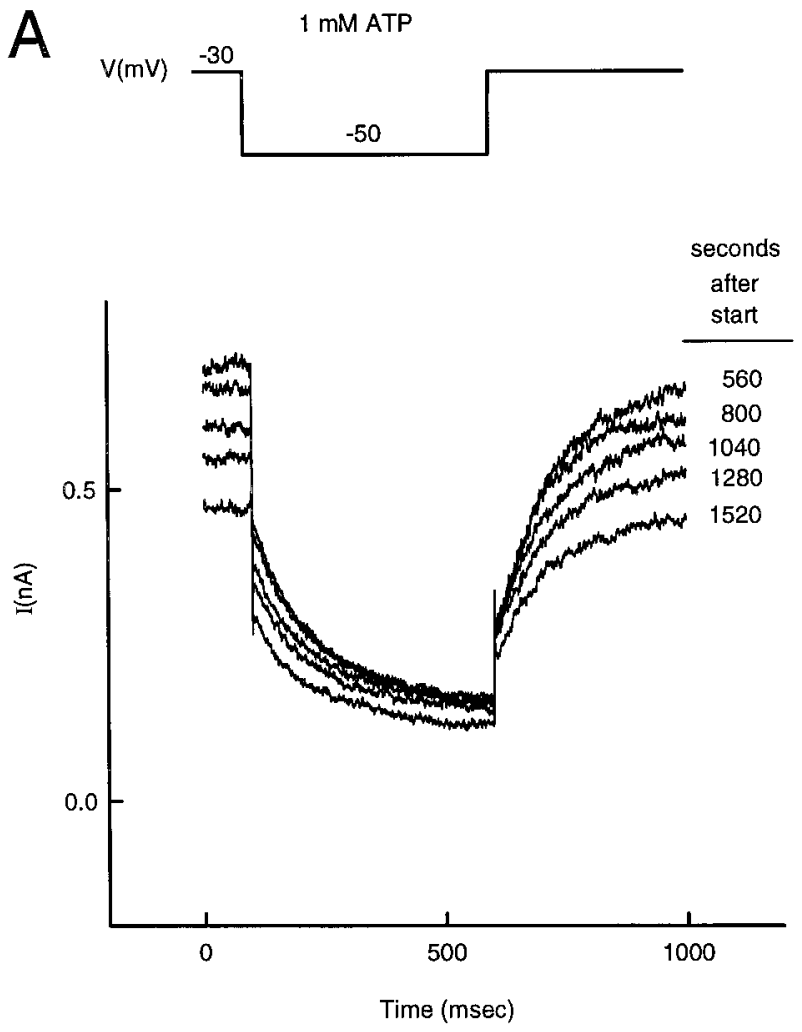

B
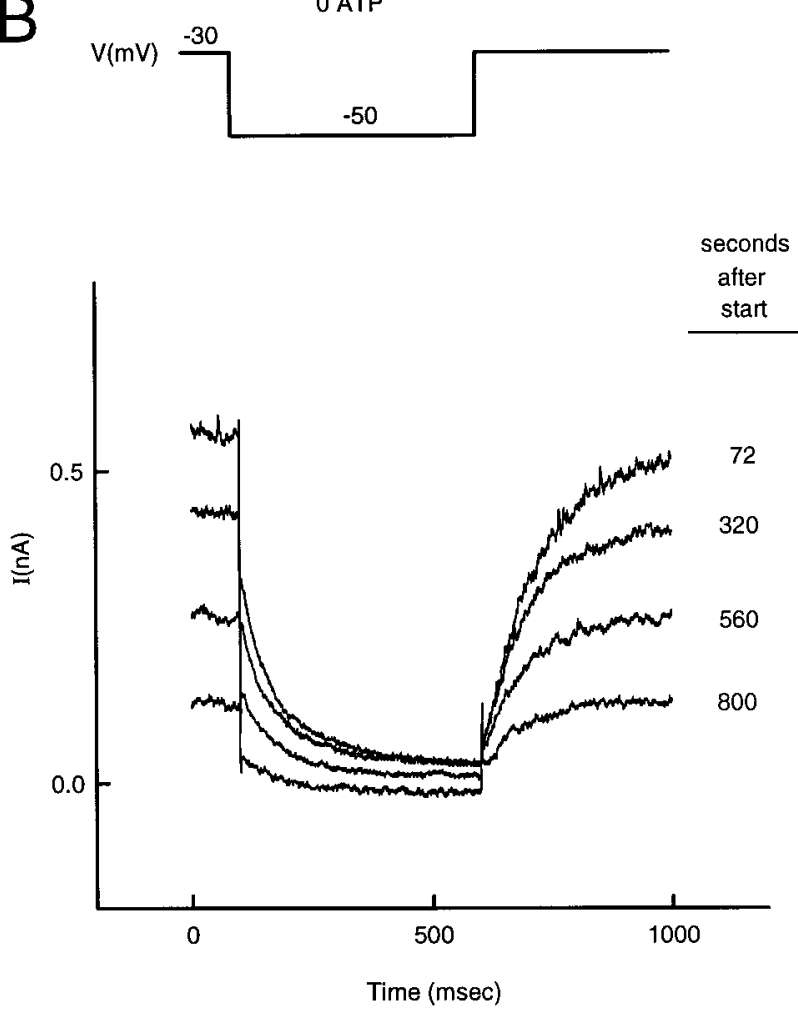

Figure 1. $I_{\mathrm{M}}$ relaxations and time-dependent changes in $I_{\mathrm{M}}$ during a whole-cell recording in the presence and absence of ATP. A, B, The top panels illustrate the voltage-clamp protocol, whereas the bottom panels show the resultant current tracings. $A$, Tracings of $I_{\mathrm{M}}$ at various times after beginning a whole-cell recording with $1 \mathrm{~mm} \mathrm{MgATP}$ in the pipette. $B$, Tracings of $I_{\mathrm{M}}$ without ATP in the pipette. Note that $I_{\mathrm{M}}$ decreases more rapidly with time in $B$ than in $A$.

with electrodes with resistances of $0.25-1 \mathrm{M} \Omega$ when filled with intracellular solution. The extracellular solution was controlled by a single-cell superfusion system as described previously (Simmons and Mather, 1991). In selected experiments, intracellular solutions were changed by perfusing the interior of the whole-cell recording electrode as described by Fischmeister and Hartzell (1987). The recordings were filtered at $1 \mathrm{kHz}$ and stored on magnetic tape.

Measurement of $\mathrm{I}_{M}$. The membrane potential was maintained at a holding potential of $-30 \mathrm{mV}$. Every $8 \mathrm{sec}$ the voltage was stepped to -50 $\mathrm{mV}$ for $500 \mathrm{msec}$ followed by return to $-30 \mathrm{mV}$. After a voltage step from -30 to $-50 \mathrm{mV}$, the outward current decreases because of the time- and voltage-dependent closing of M-channels (Fig. 1) (Brown and Adams, 1980). After returning to -30 from $-50 \mathrm{mV}$, the outward current returns exponentially (Fig. 1). The amplitude of this exponential relaxation of current is termed $I_{\mathrm{M}}(-30)$ and provides a measure of $I_{\mathrm{M}}$ that is independent of changes in other currents or "leak" currents (Simmons et al., 1994). Instantaneous and steady-state current amplitudes at -30 and -50 $\mathrm{mV}$ were determined by computer.

Quantification of the rundown of $\mathrm{I}_{M}$. To quantify the time-dependent changes in the amplitude of $I_{\mathrm{M}}$ during an experiment, the current was measured every $8 \mathrm{sec}$, and the amplitude of $I_{\mathrm{M}}(-30)$ was plotted versus time. The initial current is defined as the maximum current reached after obtaining the whole-cell configuration. The rundown of $I_{\mathrm{M}}$ under various experimental conditions is expressed as the time it took for the amplitude of $I_{\mathrm{M}}$ to decrease to $25 \%$ of this initial current value, $T(25 \%)$. If the current did not reach $25 \%$ of the initial value after $1 \mathrm{hr}$, the experiment was halted, and the $T(25 \%)$ for that cell assigned a value of $60 \mathrm{~min}$.

Solutions for electrophysiology. Composition of solutions is in $\mathrm{mm}$ unless otherwise noted. Extracellular solution: $\mathrm{NaCl} 128, \mathrm{KCl} 2.4, \mathrm{CaCl}_{2}$ 1.8, $\mathrm{MgCl}_{2}$ 1.8, HEPES 10, and TTX $0.0003, \mathrm{pH} 7.4$ (with $\mathrm{NaOH}$ ). Dissociation medium: $\mathrm{NaCl} 98, \mathrm{KCl} 2.4, \mathrm{NaH}_{2} \mathrm{PO}_{4} 0.6, \mathrm{MgCl}_{2}$ 1.8, sodium pyruvate 5, creatine 5.7, glucose 5, M199 $10 \mathrm{ml} / \mathrm{l}$, penicillin 100 $\mathrm{U} / \mathrm{ml}$, streptomycin $100 \mu \mathrm{g} / \mathrm{ml}$, and HEPES 20, pH 7.4 (with $\mathrm{NaOH}$ ). Growth medium: $\mathrm{NaCl} 118, \mathrm{KCl} 2.4$, creatine 5.7, glucose 5, sodium pyruvate 5, 100× MEM vitamins $10 \mathrm{ml} / \mathrm{l}$, penicillin $100 \mathrm{U} / \mathrm{ml}$, streptomycin $100 \mu \mathrm{g} / \mathrm{ml}, 50 \times$ MEM essential amino acids $20 \mathrm{ml} / \mathrm{L}, 100 \times \mathrm{MEM}$ nonessential amino acids $10 \mathrm{ml} / \mathrm{l}$, bovine serum albumin $1 \mathrm{mg} / \mathrm{ml}$, and HEPES 20, pH 7.4 (with $\mathrm{NaOH}$ ). Intracellular (or recording electrode) solution: KCl 120, BAPTA 1, and HEPES 10, pH 6.8 (with KOH), plus $\mathrm{MgCl}_{2}$ and nucleotides.

The amounts of $\mathrm{MgCl}_{2}$ and nucleotides to be added to the pipette solutions were determined by a computer program developed by Robert E. Godt (Medical College of Georgia, Augusta, GA) (Godt and Lindley, 1982) with modifications by H. Criss Hartzell (Emory University, Atlanta, GA). Calculations were made to give a free $[\mathrm{Mg}]$ of $0.5 \mathrm{~mm}$ and the desired concentrations of $\mathrm{MgATP}$. This program takes into account the stability constants of the various components in a manner similar to that described in Fabiato (1988). In addition to accounting for the concentrations of the various constituents, temperature, $\mathrm{pH}$, and ionic strength are also considered in the calculations. The stability constants for ATP and ADP analogs were taken to be the same as for ATP and ADP, respectively.

Most chemicals were obtained from Sigma Chemical (St. Louis, MO) or Fisher Scientific (Pittsburgh, PA). Proteolytic enzymes, nucleotides, and leupeptin were obtained from Boehringer Mannheim (Indianapolis, IN).

Measurement of calcineurin activity. Calcineurin phosphatase activity was assayed by measuring the release of para-nitrophenol from paranitrophenyl phosphate spectrophotometrically at $405 \mathrm{~nm}$ (Pallen and Wang, 1983) in a Vmax kinetic microplate reader from Molecular Devices Corp. (Menlo Park, CA). The assay was performed at $30^{\circ} \mathrm{C}$ in a 200 $\mu \mathrm{l}$ reaction volume containing $2 \mu \mathrm{g}$ of purified bovine brain calcineurin (Upstate Biotechnologies, Lake Placid, NY) and $500 \mathrm{~nm}$ purified bovine brain calmodulin (Sigma) in buffer consisting of (in mM): $120 \mathrm{KCl}, 10$ HEPES, 2 dithiothreitol, and 0.35 ATP, $\mathrm{pH}$ 8.1. Varying amounts of $\mathrm{MgCl}_{2}$ and adenine nucleotides were added according to the method described above to give buffers containing (in mM): 0.3 ADP, 3 ADP, 1 AMP-PNP, 3 AMP-PNP, or 3 sodium pyrophosphate. Blank wells were prepared containing (in $\mathrm{mM}$ ) $120 \mathrm{KCl}, 0.8 \mathrm{MgCl}_{2}$, and $10 \mathrm{HEPES}, \mathrm{pH}$ 8.1. To monitor background activity, control wells were prepared that contained buffer solutions without calcineurin and calmodulin. The microtiter plate was equilibrated for $5 \mathrm{~min}$ at $30^{\circ} \mathrm{C}$ in a precision convection oven. The reaction was initiated by the addition of $20 \mathrm{~mm}$ 


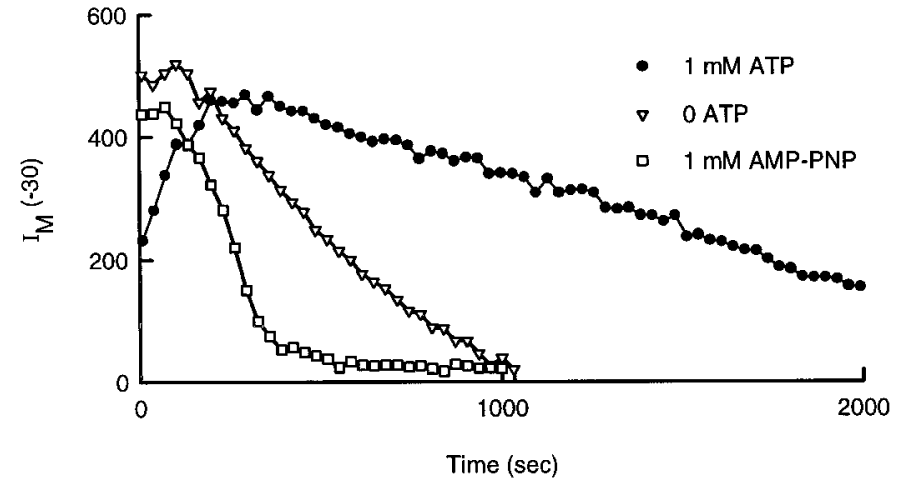

Figure 2. Plot of $I_{\mathrm{M}}$ amplitude as a function of time with and without intracellular ATP and AMP-PNP. Filled circles, ATP was included in the pipette solution to provide $1 \mathrm{~mm}$ MgATP. Open triangles, No ATP was added to the pipette solution. Open squares, AMP-PNP was included in the pipette solution to provide $1 \mathrm{~mm}$ MgAMP-PNP.

para-nitrophenyl phosphate (Sigma). After incubation at $30^{\circ}$ for $60 \mathrm{~min}$, the absorbance values for each well were measured and the background value subtracted to obtain the final optical density values.

Data analysis and statistics. Data are expressed as mean \pm SD. Tests for differences among experimental groups were determined by one-way or two-way ANOVA as appropriate followed by Student-Newman-Keuls test for multiple comparisons.

\section{RESULTS}

\section{Dependence of $I_{\mathrm{M}}$ on intracellular ATP}

Typical cellular levels of MgATP are in the range of $1 \mathrm{~mm}$. Previous studies of $I_{\mathrm{M}}$ have typically used solutions in the recording electrode that would yield approximately this amount of MgATP. The time-dependent changes in $I_{\mathrm{M}}$ observed throughout a recording period with this concentration of $\operatorname{MgATP}(1 \mathrm{mM})$ or without ATP in the recording pipette are shown in Figure 1. In this experiment, when the electrode contained $1 \mathrm{~mm}$ MgATP, the current ran down $43 \%$, from a peak of 460 to $265 \mathrm{pA}$ in the first 25 min after the start of whole-cell recording (Fig. 1A). When ATP was omitted from the pipette solution, $I_{\mathrm{M}}$ declined more rapidly (Fig. $1 B$ ), decreasing $81 \%$ from 520 to $100 \mathrm{pA}$ within $\sim 13$ min. The amplitude of $I_{\mathrm{M}}(-30)$ for the duration of these two experiments is plotted as a function of time in Figure 2. This graph shows that when ATP (1 mM) was included in the pipette (Fig. 2, filled circles), the maintenance of $I_{\mathrm{M}}$ was prolonged compared with the amplitude of $I_{\mathrm{M}}$ when ATP was omitted from the pipette (Fig. 2, open triangles). In 12 cells tested with $1 \mathrm{~mm}$ MgATP in the pipette, the time it took to decline to $25 \%$ of the initial current level, the $T(25 \%)$, was $39.0 \pm 19.0 \mathrm{~min}$. In 10 cells with no ATP added, the $T(25 \%)$ was $12.8 \pm 3.5 \mathrm{~min}$.

\section{Maintenance of $I_{\mathrm{M}}$ requires ATP hydrolysis}

There are two obvious mechanisms by which ATP may be used to prevent rundown of $I_{\mathrm{M}}$ : ATP could serve as a phosphate donor in a phosphorylation reaction that maintains the M-current, or the energy from ATP hydrolysis could be required to maintain $I_{\mathrm{M}}$. Either of these mechanisms would require the availability of a hydrolyzable form of ATP. To determine whether hydrolyzable ATP was required to maintain $I_{\mathrm{M}}$, the ATP in the pipette was replaced by AMP-PNP, a nonhydrolyzable ATP analog. $I_{\mathrm{M}}$ disappeared more rapidly when the electrode contained MgAMPPNP at a concentration of $1 \mathrm{~mm}$ (Fig. 2, open squares) than when it contained MgATP (1 mM) (Fig. 2, filled circles). The average $T(25 \%)$ with $1 \mathrm{~mm}$ MgAMP-PNP in the cell was $8.9 \pm 5.3 \mathrm{~min}$

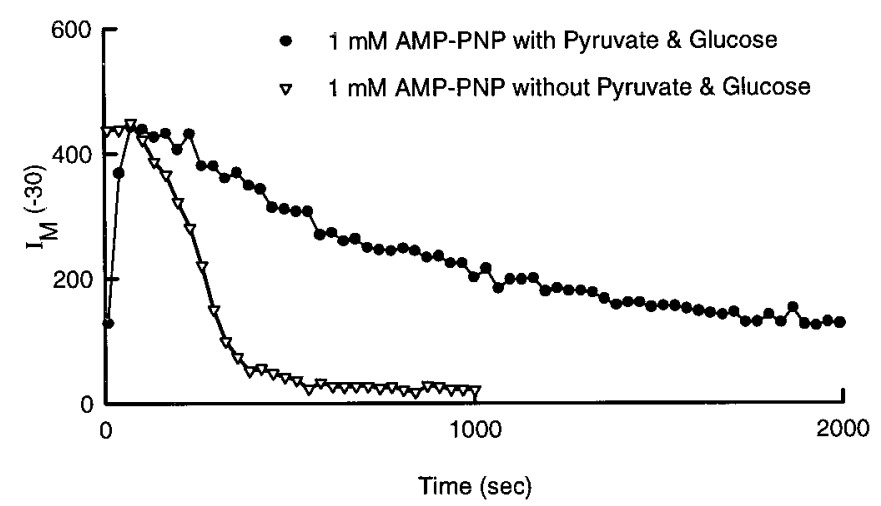

Figure 3. Extracellular metabolic substrates rescue $I_{\mathrm{M}}$ from the rundown produced by intracellular AMP-PNP. With no glucose and pyruvate in the extracellular solution and AMP-PNP in the pipette (open triangles), $I_{\mathrm{M}}$ decreased in a few hundred seconds. When glucose and pyruvate $(5$ $\mathrm{mM}$ each) were added to the extracellular medium with AMP-PNP in the pipette, rundown was slowed (filled circles).

$(n=6)$. Increasing the intracellular concentration of AMP-PNP to $3 \mathrm{~mm}$ resulted in a rapid rundown of the current with an average $T(25 \%)$ of $7.6 \pm 3.5 \min (n=20)$.

\section{Extracellular glucose and pyruvate are equivalent to intracellular ATP}

The finding that AMP-PNP resulted in a rapid disappearance of $I_{\mathrm{M}}$ was in contrast to our earlier finding that $I_{\mathrm{M}}$ was maintained at $84 \%$ of control after 13 min when AMP-PNP replaced ATP in the pipette (Simmons et al., 1990). In the previous study, $5 \mathrm{~mm}$ pyruvate and $5 \mathrm{~mm}$ glucose had been included in the extracellular solution, whereas in the present study glucose and pyruvate were omitted. Thus, it was possible that in our earlier studies the cells were utilizing the available pyruvate and glucose to synthesize enough ATP to maintain $I_{\mathrm{M}}$ in the presence of intracellular AMP-PNP. To test this possibility, glucose and pyruvate were added to the bath solution, and the effect of AMP-PNP was reexamined. When glucose and pyruvate (5 $\mathrm{mM}$ each) were present, the decrease in $I_{\mathrm{M}}$ observed with $1 \mathrm{~mm}$ AMP-PNP in the cell was slowed from a $T(25 \%)$ of $8.9 \pm 5.3 \mathrm{~min}(n=6)$ to a $T(25 \%)$ of $21.2 \pm 14.7 \mathrm{~min}(n=7)$ (Fig. 3$)$.

When either one of these energy substrates alone was added to the extracellular perfusate, the decrease of $I_{\mathrm{M}}$ with time was intermediate between that seen with both present and that seen with neither present. With $5 \mathrm{~mm}$ glucose the $T(25 \%)$ was $11.3 \pm$ $5.2 \mathrm{~min}(n=7)$, and with $5 \mathrm{~mm}$ pyruvate the $T(25 \%)$ was $11.7 \pm$ $5.9 \min (n=6)$. With pyruvate and 2-deoxy-D-glucose the $T(25 \%)$ was similar to the result seen with pyruvate alone $(11.2 \pm 3.4 \mathrm{~min}$; $n=13)$.

\section{Concentration-effect curve for ATP}

To determine the dependence of $I_{\mathrm{M}}$ on the concentration of intracellular ATP, electrodes were filled with solutions containing different amounts of MgATP. The T(25\%) with various [MgATP] in the pipette is plotted in Figure 4 (open circles). The maintenance of $I_{\mathrm{M}}$ was prolonged as [MgATP] was increased from 0 to $1 \mathrm{mM}$. With a further increase of [MgATP] to $3 \mathrm{~mm}$, however, the decrease of $I_{\mathrm{M}}$ with time was unchanged.

\section{A combination of intracellular AMP-PNP with intracellular ATP slows the rundown of $I_{M}$}

In contrast to the findings with AMP-PNP alone, when AMPPNP and ATP were combined in the pipette a different story 


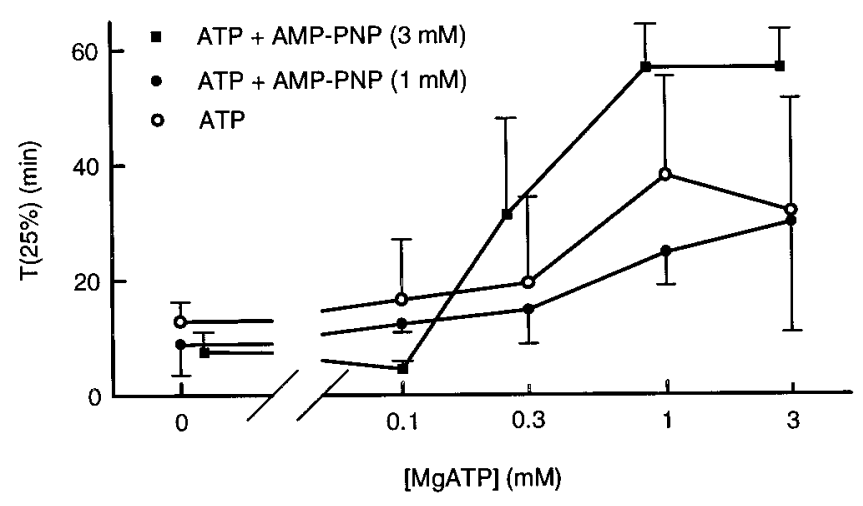

Figure 4. Dose-response curves for the effects of ATP with various concentrations of AMP-PNP. Open circles, ATP alone in the pipette; $n=$ 9-12 for each point. Filled circles, ATP plus $1 \mathrm{mM}$ AMP-PNP in the pipette; $n=4-8$ for each point. Filled squares, ATP plus 3 mM AMP-PNP in the pipette; $n=5-20$ for each point. Two-way ANOVA showed that the effect of ATP was significant $(p<0.0001)$, the effect of AMP-PNP was significant $(p<0.0002)$, and there was a significant interaction between the effects of ATP and AMP-PNP $(p<0.0001)$.

emerged. In Figure 4 the filled symbols show the concentrationeffect curve for MgATP in the presence of a constant concentration of $1 \mathrm{~mm}$ MgAMP-PNP ( filled circles) or $3 \mathrm{~mm} \mathrm{MgAMP-PNP}$ ( filled squares).

The concentration-effect curve for ATP exhibited a downward shift in the presence of $1 \mathrm{~mm}$ MgAMP-PNP. At a concentration of $3 \mathrm{~mm}$, however, MgAMP-PNP in the simultaneous presence of MgATP at concentrations of $\geq 0.3 \mathrm{~mm}$ resulted in a sustained maintenance of $I_{\mathrm{M}}$. With $1 \mathrm{~mm} \mathrm{MgATP}$ and $3 \mathrm{~mm}$ MgAMP-PNP, there was essentially no rundown of the current during a $1 \mathrm{hr}$ recording period. This compares to a rundown to $25 \%$ in $39 \mathrm{~min}$ with $1 \mathrm{~mm}$ MgATP alone or in $7.6 \mathrm{~min}$ with $3 \mathrm{~mm}$ MgAMP-PNP alone.

The rapid rundown observed with $3 \mathrm{~mm}$ AMP-PNP could be reversed by intracellular perfusion of $1 \mathrm{~mm}$ ATP along with $3 \mathrm{~mm}$ AMP-PNP. This experiment was repeated in five cells. In all cases the rundown normally observed with $3 \mathrm{mM} \mathrm{AMP-PNP}$ was halted when ATP was perfused intracellulary. In four of the five cells the current rundown was not only halted but was also reversed, as illustrated in Figure 5.

These results suggest that there are dual effects of adenine nucleotides on $I_{\mathrm{M}}$. Maintenance of $I_{\mathrm{M}}$ requires a hydrolyzable form of ATP, because a nonhydrolyzable analog alone is insufficient to maintain $I_{\mathrm{M}}$. In the presence of ATP, $I_{\mathrm{M}}$ is further regulated by the simultaneous presence of nonhydrolyzable adenine nucleotides.

To see whether other nucleotides or analogs might be more effective than AMP-PNP in producing this effect, we have tested the effects of a variety of other nucleotide analogs on $I_{\mathrm{M}}$.

\section{Effects of adenine nucleoside diphosphate analogs on the rundown of $I_{M}$}

Concentration-effect curves for MgATP have been constructed in the presence of a constant concentration of ADP or AMP-CP (3 mM). In these experiments, $\mathrm{Mg}$ and ATP were added at concentrations calculated to provide $0.5 \mathrm{~mm}$ free $\mathrm{Mg}$ and the desired amount of MgATP. Of the $3 \mathrm{~mm}$ added ADP or AMP$\mathrm{CP}, 1 \mathrm{~mm}$ is predicted to be free ion, $0.8 \mathrm{~mm}$ complexed to $\mathrm{Mg}$ ions, and the remainder complexed to other metals. Figure 6 shows a comparison of the effects of ATP alone (open circles) and in the presence of $3 \mathrm{~mm}$ ADP (open triangles) or $3 \mathrm{~mm} \mathrm{AMP-CP}$

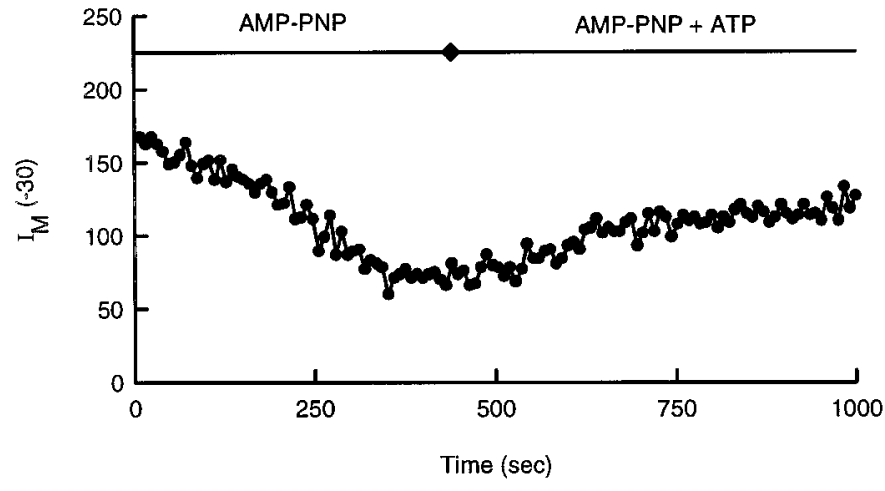

Figure 5. Reversal of rundown of $I_{\mathrm{M}}$ produced by intracellular AMPPNP by intracellular perfusion with ATP. A whole-cell recording from a single sympathetic neuron was inititated at time 0 with an electrode containing $3 \mathrm{~mm}$ MgAMP-PNP and 0 ATP. As typically observed with AMP-PNP, the current ran down. At the time indicated by the diamond, the solution perfusing the whole-cell recording electrode was changed to one containing $3 \mathrm{~mm}$ AMP-PNP plus $1 \mathrm{~mm}$ ATP. Addition of ATP intracellularly halted and partially reversed the rundown produced by AMP-PNP alone.

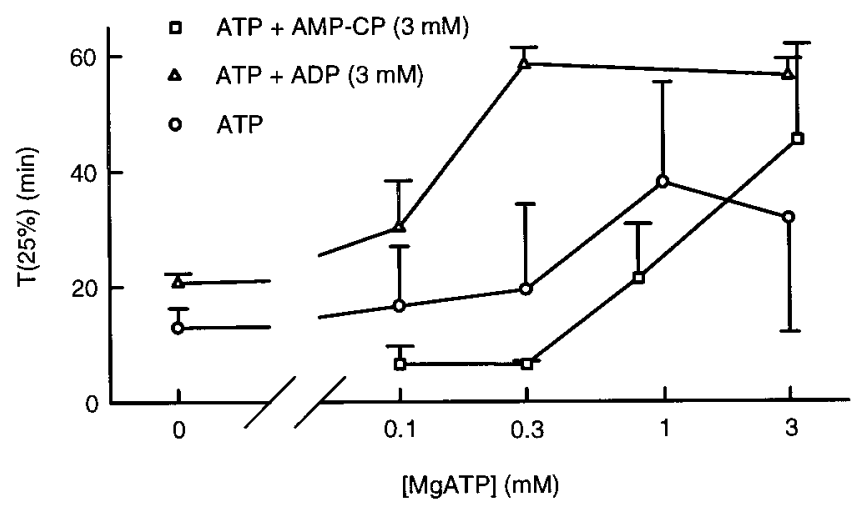

Figure 6. Dose-response curves for the effects of ATP in the presence of ADP and an ADP analog. Open circles, ATP alone in the pipette; $n=$ 9-12 for each point. Open triangles, ATP plus $3 \mathrm{~mm}$ ADP in the pipette; $n=3-4$ for each point. Open squares, ATP plus $3 \mathrm{mM} \mathrm{AMP-CP}$ in the pipette; $n=3-5$ for each point. Two-way ANOVA for the effects of ATP and ADP showed that the effect of ATP was significant $(p<0.0001)$, the effect of ADP was significant $(p<0.001)$, and the significance of the interaction between ATP and ADP was borderline $(p=0.054)$. Analysis of the effects of ATP and AMP-CP showed that the effect of ATP was significant $(p<0.0002)$, the effect of AMP-CP was not significant ( $p>$ $0.05)$, and the significance of the interaction between ATP and AMP-CP was borderline $(p=0.072)$.

(open squares). The ability of ATP to slow the rundown of $I_{\mathrm{M}}$ was enhanced in the simultaneous presence of ADP. In contrast to this, the ability of ATP to slow the rundown of $I_{\mathrm{M}}$ was inhibited by $3 \mathrm{~mm}$ AMP-CP for ATP concentrations of $\leq 1 \mathrm{~mm}$ but was enhanced when ATP was present at a concentration of $3 \mathrm{~mm}$. A concentration-effect curve for ADP in the presence of $0.3 \mathrm{~mm}$ ATP is shown in Figure 7.

From the dose-response curves shown, it is apparent that the ability of compounds to alter the rundown of $I_{\mathrm{M}}$ is most easily observed with $0.3 \mathrm{~mm} \mathrm{MgATP}$ in the pipette. With $0.3 \mathrm{~mm}$ MgATP, the $T(25 \%)$ for AMP-CP (3 mM) was $6.4 \mathrm{~min}$, for AMP-PNP (1 mM) was $14.7 \mathrm{~min}$, for MgATP alone was $19.4 \mathrm{~min}$, for AMP-PNP (3 mM) was $31.2 \mathrm{~min}$, and for ADP (3 mM) was $58.3 \mathrm{~min}$. The actions of a variety of other analogs on $I_{\mathrm{M}}$ rundown in the presence of $0.3 \mathrm{~mm} \mathrm{MgATP}$ have also been tested. The 


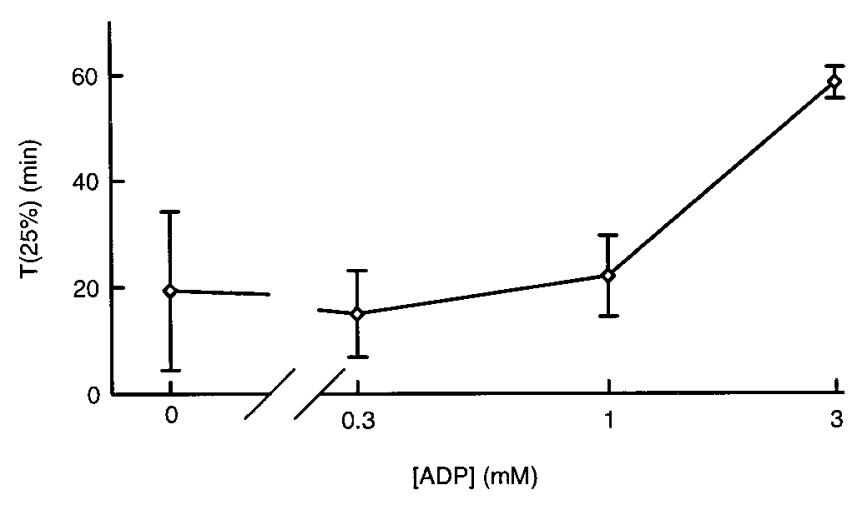

Figure 7. Dose-response curve for various concentrations of ADP to maintain $I_{\mathrm{M}}$ in the presence of a constant concentration of $0.3 \mathrm{~mm}$ MgATP; $n=11$ at 0 ADP and 4 at the other points. The effect of ADP on the $T(25 \%)$ was statistically significant $(p<0.0002)$.

\begin{tabular}{lccr}
\hline $\begin{array}{l}\text { Table 1. Effects of various nucleotides and nucleotide analogs on run } \\
\text { down of } \mathbf{I}_{\mathbf{M}}\end{array}$ & & & \\
& & & \\
& T $(25 \%)$ & $(\mathrm{min})$ & \\
\cline { 2 - 4 } Condition & 58.3 & 2.9 & 4 \\
\hline 3 mM ADP + 0.3 mM ATP & 51.7 & 17.6 & 4 \\
3 mM sodium pyrophosphate + 0.3 mM ATP & 32.4 & 16.7 & 3 \\
3 mM UDP + 0.3 mM ATP & 31.2 & 16.8 & 5 \\
3 mM AMP-PNP + 0.3 mM ATP & 26.2 & 10.0 & 3 \\
3 mM ADP + 0.3 mM ATP, 0 Mg & 24.7 & 19.4 & 4 \\
3 mM AMP + 0.3 mM ATP & 19.4 & 14.9 & 11 \\
0.3 mM ATP & 19.0 & 8.7 & 4 \\
3 mM CDP + 0.3 mM ATP & 14.9 & 5.0 & 4 \\
3 mM sodium pyrophosphate + 0 ATP & 14.7 & 5.8 & 8 \\
1 mM AMP-PNP + 0.3 mM ATP & 14.1 & 2.8 & 6 \\
3 mM AMP-PCP + 0.3 mM ATP & 13.9 & 5.6 & 4 \\
3 mM IDP + 0.3 mM ATP & 9.8 & 2.5 & 3 \\
3 mM GDP + 0.3 mM ATP & 6.6 & 4.2 & 4 \\
3 mM cAMP + 0.3 mM ATP & 6.4 & 0.6 & 3 \\
3 mM AMP-CP + 0.3 mM ATP & &
\end{tabular}

$T(25 \%)$ for these compounds is shown in Table 1 . The data are listed in order of decreasing $T(25 \%)$.

\section{Effects of adenine nucleotides and analogs on other electrophysiological properties}

In addition to measuring $I_{\mathrm{M}}$, several other parameters have been measured to examine the specificity of the effects with respect to $I_{\mathrm{M}}$. For statistical comparisons, $t$ tests have been made between the data obtained under the various experimental conditions to the data obtained with $0.3 \mathrm{~mm}$ ATP. When recording $I_{\mathrm{M}}$ a run-up of current is sometimes observed during the first $30-60 \mathrm{sec}$ of recording (Figs. 2, 3). The time from obtaining whole-cell recording to reaching this initial peak current was not altered by the different nucleotides. Nor was the amplitude of $I_{M}$ at this initial time point affected. The leak current at this time and at the $T(25 \%)$ were also measured and did not differ from the values obtained with $0.3 \mathrm{~mm}$ ATP.

\section{Calcineurin activity}

It has been shown previously that the activity of the calcium- and calmodulin-dependent phosphatase 2B calcineurin modulates $I_{\mathrm{M}}$ in neurons from rat sympathetic ganglia (Marrion, 1996). It has
Table 2. Effects of various nucleotides and nucleotide analogs on calcineurin phosphatase activity

\begin{tabular}{lll} 
& \multicolumn{2}{l}{ OD } \\
\cline { 2 - 3 } Condition & Mean & SD \\
\cline { 2 - 3 } $3 \mathrm{mM}$ sodium pyrophosphate $+0.3 \mathrm{mM}$ ATP & $0.02^{*}$ & 0.03 \\
\hline $3 \mathrm{mM}$ AMP-PNP + 0.3 mM ATP & $0.23^{*}$ & 0.10 \\
$3 \mathrm{mM}$ ADP + 0.3 mM ATP & $0.30^{*}$ & 0.09 \\
\hline $0.3 \mathrm{mM}$ ATP & 0.54 & 0.17 \\
$0.3 \mathrm{mM}$ ADP $+0.3 \mathrm{mM}$ ATP & 0.60 & 0.35 \\
\hline $1 \mathrm{mM}$ AMP-PNP $+0.3 \mathrm{mM}$ ATP & $0.95^{*}$ & 0.27
\end{tabular}

A one-way ANOVA showed significant effects of the various solutions on calcineurin activity $(p<0.001)$. Results grouped within the ruled area are not significantly different from one another.

* indicates significantly different from $0.3 \mathrm{mM}$ ATP.

$n=12$ for each point.

The assay was repeated twice with six wells under each condition.

also been shown that phosphates modulate calcineurin activity (King and Huang, 1984). To test the hypothesis that the effects of intracellular nucleotide phosphates on $I_{\mathrm{M}}$ may be accounted for by the effects of these nucleotides on calcineurin activity, the effects of concentrations of selected nucleotides that were shown to alter $I_{\mathrm{M}}$ above have been tested on calcineurin phosphatase activity. The results of the calcineurin phosphatase assays are shown in Table 2.

\section{Effects of a calcineurin inhibitor}

Cyclosporin A is an inhibitor of calcineurin activity (Liu et al., 1991). When applied intracellularly to single neurons with $0.3 \mathrm{~mm}$ ATP, cyclosporin A at a concentration of $500 \mathrm{~nm}$ increased the $T(25 \%)$ from $19.4 \pm 14.9$ to $30.5 \pm 10.7$ and to $41.9 \pm 15.8 \mathrm{~min}$ when added at a concentration of $1 \mu \mathrm{M}$. Cyclosporin A (500 nM) had no effect when applied outside the cell $[T(25 \%)=9.1 \pm$ $1.8, n=2]$.

\section{DISCUSSION}

The results clearly show that nucleotide phosphates play a critical role in the regulation of $I_{\mathrm{M}}$. Furthermore, there are at least two ways by which adenine nucleotides can modulate $I_{\mathrm{M}}$. First, hydrolyzable ATP must be available for $I_{M}$ to be maintained. Second, above and beyond this requirement for a basal level of ATP, other nucleotide phosphates and analogs act to further modulate the level of $I_{\mathrm{M}}$. These results are consistent with regulation of $I_{\mathrm{M}}$ by kinases and phosphatases.

Several previous studies have suggested that $I_{M}$ is regulated by phosphorylation catalyzed by myosin light chain kinase. Application of myosin light chain kinase inhibitors decreases $I_{\mathrm{M}}$, whereas intracellular perfusion of catalytic subunits of myosin light chain kinase enhances $I_{M}$ (Akasu et al., 1993; Tokimasa et al., 1995). The result presented here, that ATP is required to prevent the rundown of $I_{\mathrm{M}}$, extends these findings to suggest that myosin light chain kinase-catalyzed phosphorylation events not only alter the amplitude of $I_{\mathrm{M}}$ but may be required for the maintenance of $I_{\mathrm{M}}$.

In addition to the positive regulation of $I_{\mathrm{M}}$ by myosin light chain kinase-catalyzed phosphorylation events, $I_{\mathrm{M}}$ has also been shown to be negatively regulated by phosphatase-catalyzed dephosphorylation events (Marrion, 1996). Marrion (1996) showed that $I_{\mathrm{M}}$ was decreased when a catalytically active form of calcineurin was applied intracellularly to rat sympathetic neurons. This suggests that $I_{\mathrm{M}}$ is regulated by the calcium and calmodulin- 
dependent phosphatase type $2 \mathrm{~B}$, calcineurin, and that this phosphatase activity results in a decrease in the amplitude of $I_{\mathrm{M}}$.

In the present study, several compounds have been shown to prevent the rundown of $I_{\mathrm{M}}$. These compounds include ADP, $\mathrm{PP}_{\mathrm{i}}$, and cyclosporin $\mathrm{A}$. The results of the phosphatase assay show that ADP and $\mathrm{PP}_{\mathrm{i}}$, at concentrations that prevent the rundown of $I_{\mathrm{M}}$, are effective inhibitors of calcineurin phosphatase activity. Cyclosporin A is well established as a calcineurin inhibitor (Liu et al., 1991). These data support the previous findings that $I_{M}$ is regulated by phosphatase activity (Marrion, 1996) and further illustrate that this phosphatase activity can be regulated by endogenous compounds such as ADP and $\mathrm{PP}_{\mathrm{i}}$. Pyrophosphate and ADP at a concentration of $3 \mathrm{~mm}$ significantly inhibited calcineurin phosphatase activity and slowed $I_{\mathrm{M}}$ rundown. A $0.3 \mathrm{~mm}$ concentration of ADP changed neither calcineurin activity nor the rundown of $I_{\mathrm{M}}$.

This conclusion is further supported by the results obtained with AMP-PNP. The effect of AMP-PNP depended on both its concentration and on the concentration of ATP present. As intracellular ATP was increased, the maintenance of $I_{\mathrm{M}}$ was prolonged. When AMP-PNP at a concentration of $1 \mathrm{~mm}$ was added along with ATP, the ability of ATP to maintain $I_{\mathrm{M}}$ was decreased. When AMP-PNP at a concentration of $3 \mathrm{~mm}$ was added along with ATP at concentrations of $\geq 0.3 \mathrm{~mm}$, however, the maintenance of $I_{\mathrm{M}}$ was enhanced. These results can be interpreted in accordance with the effects of AMP-PNP on calcineurin activity. At a concentration of $1 \mathrm{mM}$, AMP-PNP increased calcineurin activity and speeded $I_{M}$ rundown. At $3 \mathrm{~mm}$, AMP-PNP decreased calcineurin activity and slowed $I_{\mathrm{M}}$ rundown.

Although there is qualitative agreement between the effects of the various nucleotides in the calcineurin assays and their effects on $I_{\mathrm{M}}$ rundown, there are quantitative differences. For example, 3 mM ADP and $3 \mathrm{~mm}$ AMP-PNP have equivalent, less than $\mathrm{PP}_{\mathrm{i}}$, effects in the calcineurin assay. On $I_{\mathrm{M}}$ rundown, the effect of ADP is greater than that of $\mathrm{PP}_{i}$, whereas the effect of AMP-PNP is less than $\mathrm{PP}_{\mathrm{i}}$. This difference might be accounted for by the different situations under which these two measurements were made. The bovine brain calcineurin in vitro is clearly under different conditions than the calcineurin in a bullfrog sympathetic ganglion cell undergoing whole-cell recording.

\section{Importance of considering the role of pyruvate and glucose in physiological solutions on ionic currents}

Many studies of ionic currents in single cells use whole-cell recordings. With this technique, it is generally assumed that the electrode contents effectively suffuse the cell and thereby control the cellular contents. Metabolic substrates such as glucose and pyruvate are routinely added to either the extracellular medium or to the pipette solution during such experiments. The data presented here demonstrate that these additives can have important modulatory effects on ionic currents. These effects may occur even when the pyruvate and glucose are only added extracellularly and recordings are made with large electrodes that would be expected to provide good control of intracellular constituents.

The ability of extracellularly applied pyruvate and glucose to substitute for intracellular ATP shows that enzymatically dissociated single neurons maintain active metabolic processes. The $T(25 \%)$ in the presence of extracellular pyruvate and glucose and intracellular AMP-PNP was 21.2 min. A similar T(25\%) of 24.6 min was obtained with $1 \mathrm{~mm}$ MgATP and $1 \mathrm{~mm} \mathrm{AMP-PNP}$. Thus, adding $5 \mathrm{~mm}$ glucose and $5 \mathrm{~mm}$ pyruvate to the extracellular medium is equivalent to putting $1 \mathrm{~mm} \mathrm{MgATP}$ in the pipette.

This can account for some of the discrepancies observed in previous work from different laboratories. Those studies that had reported $I_{\mathrm{M}}$ was maintained without ATP had $5 \mathrm{~mm}$ pyruvate and $5 \mathrm{~mm}$ glucose in the extracellular solution (Simmons et al., 1990) or $10 \mathrm{~mm}$ glucose in the extracellular solution and $10 \mathrm{~mm}$ glucose in the pipette solution (Chen and Smith, 1992). Those studies that had reported that $I_{M}$ disappeared in the absence of ATP (Pfaffinger, 1988; Tokimasa and Akasu, 1990) did not add either pyruvate or glucose to their solutions.

These data also show that ATP is required for the maintenance of $I_{\mathrm{M}}$ as suggested previously (Pfaffinger 1988; Tokimasa and Akasu 1990). The finding that the $M$ current was maintained in the presence of intracellular ATP, but not with AMP-PNP alone, supports previous work showing modulation of $I_{\mathrm{M}}$ by kinases and phosphatases (Akasu et al., 1993; Marrion, 1996).

In conclusion, the data presented here show that the levels of endogenous compounds such as ATP, ADP, and $\mathrm{PP}_{\mathrm{i}}$ play an important role in modulating the level of $I_{M}$ in single neurons. The present results, when taken in perspective with other studies on $I_{\mathrm{M}}$, are consistent with a dual regulation of $I_{\mathrm{M}}$ by kinases and phosphatases. In the presence of ATP, myosin light chain kinasecatalyzed phosphorylation enhances $I_{\mathrm{M}}$. Calcineurin-catalyzed dephosphorylation inhibits $I_{\mathrm{M}}$. High levels of ADP or $\mathrm{PP}_{\mathrm{i}}$ decrease the activity of calcineurin and enhance $I_{\mathrm{M}}$. The ultimate result of an increased $I_{M}$ is a decrease in neuronal excitability. Thus, the intracellular levels of adenine nucleotides provide an important mechanism for cellular regulation of neuronal excitability by influencing the level of $I_{\mathrm{M}}$.

\section{REFERENCES}

Akasu T, Ito M, Nakano T, Schneider CR, Simmons MA, Tanaka T, Tokimasa T, Yoshida M (1993) Myosin light chain kinase occurs in bullfrog sympathetic neurons and may modulate voltage-dependent potassium currents. Neuron 11:1133-1145.

Brown DA, Adams PR (1980) Muscarinic suppression of a novel voltage-sensitive $\mathrm{K}^{+}$-current in a vertebrate neuron. Nature 283:673-676.

Brown DA, Marrion NV, Smart TG (1989) On the transduction mechanism for muscarine-induced inhibition of M-current in cultured rat sympathetic neurons. J Physiol (Lond) 413:469-488.

Chen H, Smith PA (1992) M-currents in frog sympathetic ganglion cells: manipulation of membrane phosphorylation. $\mathrm{Br} \mathrm{J}$ Pharmacol 105:329-334.

Fabiato A (1988) Computer programs for calculating total from specified free or free from specified total ionic concentrations in aqueous solutions containing multiples metals and ligands. Methods Enzymol 157:378-417.

Fischmeister RF, Hartzell HC (1987) Cyclic guanosine 3'5'monophosphate regulates the calcium current in single cells from frog ventricle. J Physiol (Lond) 387:453-472.

Godt RE, Lindley BD (1982) Influence of temperature upon contractile activation and isometric force production in mechanically skinned muscle fibers of the frog. J Gen Physiol 80:279-297.

Halliwell JV (1986) M-currents in human neocortical neurons. Neurosci Lett 67:1-6.

Halliwell JV, Adams PR (1982) Voltage-clamp analysis of muscarinic excitation in hippocampal neurons. Brain Res 250:71-92.

Hamill OP, Marty A, Neher E, Sakmann B, Sigworth FJ (1981) Improved patch-clamp techniques for high-resolution current recording from cells and cell-free membrane patches. Pflüeg Arch Eur J Physiol 391:85-100.

King MM, Huang CY (1984) The calmodulin-dependent activation and deactivation of the phosphoprotein phosphatase calcineurin and the effect of nucleotides, pyrophosphates, and divalent metal ions. J Biol Chem 259:8847-8856.

Liu J, Farmer Jr JD, Lane WS, Friedman J, Weissman I, Schreiber SL 
(1991) Calcineurin is a common target of cyclophilin-cyclosporin A and FKBP-FK506 complexes. Cell 66:807-815.

Lopez HS, Adams PR (1989) A G-protein mediates the inhibition of the voltage-dependent potassium $M$ current by muscarine, LHRH, substance P, and UTP in bullfrog sympathetic neurons. Eur J Neurosci 1:529-542.

Marrion NV (1996) Calcineurin regulates M channel modal gating in sympathetic neurons. Neuron 16:163-173.

Murase K, Ryu PD, Randic M (1986) Substance P augments a persistent slow inward calcium-sensitive current in voltage-clamped spinal dorsal horn neurons of the rat. Brain Res 365:369-376.

Pallen CJ, Wang JH (1983) Calmodulin-stimulated dephosphorylation of $p$-nitrophenyl phosphate and free phosphotyrosine by calcineurin. J Biol Chem 258:8550-8553.

Pfaffinger P (1988) Muscarine and t-LHRH suppress M current by activating an IAP-insensitive G-protein. J Neurosci 8:3343-3353.

Simmons MA, Mather RJ (1991) Selectivity of the effects of guanosine$5^{\prime}-O$-(2-thiodiphosphate) on agonist inhibition of the $\mathrm{M}$ current in amphibian sympathetic neurons. J Neurosci 11:2130-2134.

Simmons MA, Mather RJ (1992) Intracellular guanosine-5'-O-(2- thiodiphosphate) alters the dynamics of receptor-mediated responses in bullfrog sympathetic neurons. Mol Pharmacol 41:527-534.

Simmons MA, Becker JB, Mather RJ (1990) Desensitization of the inhibition of the $\mathrm{M}$ current in sympathetic neurons: effects of ATP analogs polyanions and multiple agonist applications. Neuron 4:557-562.

Simmons MA, Schneider CR, Krause JE (1994) Regulation of the responses to gonadotropin-releasing hormone, muscarine and substance $\mathrm{P}$ in sympathetic neurons by changes in cellular constitutents and intracellular application of peptide fragments of the substance P receptor. J Pharmacol Exp Ther 271:581-589.

Sims SM, Singer JJ, Walsh Jr JV (1985) Cholinergic agonists suppress a potassium current in freshly dissociated smooth muscle cells of the toad. J Physiol (Lond) 367:503-529.

Tokimasa T, Akasu T (1990) ATP regulates muscarine-sensitive potassium current in dissociated bull-frog primary afferent neurons. J Physiol (Lond) 426:241-264.

Tokimasa T, Ito M, Simmons MA, Schneider CR, Tanaka T, Nakano T, Akasu T (1995) Inhibition by wortmannin of M-current in bullfrog sympathetic neurons. Br J Pharmacol 114:289-295. 